\title{
Extrahepatic versus Intrahepatic Hilar Control for Right Hepatectomy: An Updated Experience
}

\author{
Mohammad Habib A. Ben Cresswell Kandiah Chandrakumaran \\ Fenella K.S. Welsh Timothy G. John Myrddin Rees \\ Basingstoke Hepatobiliary Unit, Basingstoke and North Hampshire NHS Foundation Trust, Basingstoke, UK
}

\section{Key Words}

Right hepatectomy • Anterior intra-hepatic approach •

Extra-glissonian stapling

\begin{abstract}
Background: Inflow control prior to transection for right hepatectomy may be achieved either by dissection and ligation of the individual hilar structures outside of the liver (EHD) or by mass stapling of the inflow structures within the hepatic parenchyma. Our preference is for the anterior intrahepatic approach (AIA) with mass stapling, in order to minimise the risk of inadvertent injury of the left-sided inflow and to preserve as much parenchyma as possible. In this paper, we present our experience over the last 10 years and compare it with results from the EHD technique. Methods: Data for a 10-year period from 2000 to 2010 were extracted retrospectively from a prospectively collected database. Results in each group were measured by a combination of technical and oncological outcomes. Groups were compared by way of descriptive statistics and differences tested for significance by appropriate statistical means. Results: 411 right hepatectomies were performed for colorectal metastases. Of these, 242 were by AIA and 169 by EHD. Both groups were well matched in demographic terms and according to disease burden, although more extended resections were performed in the EHD group. Operative duration (433 vs. 350
\end{abstract}

$\mathrm{min}$ ), blood loss (420 vs. $348 \mathrm{ml}$ ) and incidence of bile leaks (4 vs. 2) were all lower in the AIA group. All other technical and oncological outcomes were equivalent. Conclusion: The AIA approach provides equivalent morbidity, mortality and oncological outcome to the EHD dissection technique and may confer the benefits of being safer and providing greater scope to preserve hepatic parenchyma.

Copyright $\odot 2012$ S. Karger AG, Basel

\section{Introduction}

The anterior intrahepatic approach (AIA) to right hepatectomy was first described by Tung and Quang in 1963 [1], who later popularised and promoted it as the 'anterior trans-parenchymatous technique' in 1965 [2]. This built upon the previous description of a posterior approach to control of the portal pedicle by way of hepatotomy - first described by Lin et al. in 1960 [3] and presented as a safer alternative to the classic approach of extra-hepatic hilar dissection (EHD), which had been described in 1952 by Lortat-Jacob et al. [4].

In our hands, the modern AIA consists of commencing parenchymal transection in the principle plane of the liver under inflow (Pringle) occlusion, using the cavitron ultrasonic surgical aspirator [5]. This first phase of transection is continued until the right main portal inflow or

\section{KARGER}

Fax +41613061234 E-Mail karger@karger.ch www.karger.com
(C) 2012 S. Karger AG, Basel

0253-4886/12/0291-0018\$38.00/0

Accessible online at:

www.karger.com/dsu
Myrddin Rees

North Hampshire Hospital

Aldermaston Road

Basingstoke RG25 9NA (UK)

Tel. +44 1256313 558, E-Mail myrddinrees@bnhft.nhs.uk 
first order anterior and posterior branches have been dissected, following which they are divided using an endoscopic linear cutting stapler of appropriate size. Transection is then completed with division of the right hepatic vein (again using an endoscopic stapler) outside of the liver parenchyma. The AIA presents the potential benefits of reducing operative time and minimises risk of inadvertent injury of the contralateral inflow structures during EHD, and has been greatly facilitated by the advent and development of stapling devices [6].

We have previously shown that the preferential, but selective use of the AIA to right hepatectomy is both technically feasible and at least as safe as the conventional approach of EHD [7]. Our preference has been to adopt the AIA wherever possible, with tumours lying close to the porta hepatis $(<1-2 \mathrm{~cm})$ being the only absolute contraindication to this technique. We believe that this approach holds three advantages. The risk of iatrogenic injury of the left-sided structures is minimised, vascular inflow and biliary drainage of the future liver remnant is maximised, and the technique affords flexibility to safely deal with unexpected anomalies of intrahepatic portal anatomy.

With the expanding requirement for parenchymalsparing resections as the result of both increased chemotherapy-associated hepato-toxicity and planned staged liver resection, we would advocate the AIA where possible and present an updated 10-year experience with this technique.

\section{Materials and Methods}

A retrospective case-controlled study of a prospectively collected database was performed. All patients undergoing right hepatectomy for colorectal liver metastases over a 10-year period between September 2000 and 2010 were included. The choice of operative technique was not randomised and reflected the surgeon's preference with a trend towards increased use of the AIA as the series progressed. Close proximity of tumour to the portal bifurcation was the only absolute indication for EHD in the more contemporary part of the series.

The preoperative and radiological selection of patients and a detailed description of operative and anaesthetic techniques have been described previously in detail [7-9].

Transection was performed using ultrasonic dissection (cavitron ultrasonic surgical aspirator) [5] under intermittent Pringle manoeuvre, generally involving $20 \mathrm{~min}$ of occlusion alternating with $5 \mathrm{~min}$ of reperfusion. All stapling procedures were performed using the Autosuture Endovascular Device (Covidien UK, Gosport, UK).

The primary outcome measures were mortality, morbidity, oncological margins, technical factors and duration of operation.

Extrahepatic versus Intrahepatic Hilar

Control for Right Hepatectomy
Statistical analysis was performed using SPSS version 15 (SPSS, Inc, Chicago, Ill., USA). Normality of distribution was tested using Kolmogorov-Smirnov test, with age being the only normally distributed continuous variable. We therefore utilised paired t test for age, Cochran's $Q$ test for binary categorical variables and Wilcoxon's signed rank test for all continuous variables.

\section{Results}

\section{Demographics}

A total of 1,186 resections for colorectal liver metastases were performed over the study period, with 411 of these being right hepatectomy - comprising $34 \%$ of the total workload. 242 patients underwent the AIA, whilst classical EHD was performed in 169 cases.

Mean age at the time of surgery was 63 (range 29-85 years). Both groups were well matched for age, sex, ASA grade, the presence of background liver disease (table 1) and the interval between the cessation of chemotherapy and liver resection.

\section{Disease Burden}

The number, maximum size and hepatic distribution of metastases in both groups were broadly similar. Metastatic spread was confined to the right lobe in 77.2 and $74.7 \%$ of cases for AIA and EHD groups, respectively. There was no significant difference in the grade or stage of the primary colorectal lesion (table 2).

\section{Extent of Resection}

There were more extended resections performed in the EHD group (46 vs. 16\%), reflecting the need to secure a clear margin with more centrally placed disease in this group. In both groups, the right hepatectomy was accompanied by a non-anatomical resection or wedge excision from the left side in a significant proportion of patients (table 2).

\section{Duration of Operation and Hospital Stay}

The duration of operation was significantly different, with a mean of $350 \mathrm{~min}$ in the AIA group versus $433 \mathrm{~min}$ in the EHD group ( $p=0.001)$. There was no difference in hospital stay between the groups, with a median of 8 days in the AIA group (range 3-93) and 9 days in the EHD group (range 3-95).

\section{Blood Loss}

Total blood loss was significantly higher in the EHD group with a median of $420 \mathrm{ml}$ (range 75-5,344) versus $348 \mathrm{ml}$ (range $80-1,188)$ in the AIA group $(\mathrm{p}<0.001)$. 
Table 1. Background and details of study groups

\begin{tabular}{lccc}
\hline & AIA $(\mathrm{n}=242)$ & EHD $(\mathrm{n}=169)$ & p value \\
\hline Median age at operation, years & $63(29-85)$ & $64(30-84)$ & 0.959 \\
Sex & & & 0.461 \\
$\quad$ Male & $143(59.3)$ & $107(62.9)$ & 0.285 \\
Female & $99(40.7)$ & $62(37.1)$ & \\
ASA grade & & & \\
1 & $5(2.1)$ & $1(0.6)$ & 0.769 \\
2 & $191(78.8)$ & $127(75.3)$ & \\
3 & $45(18.7)$ & 0 & \\
4 & $1(0.4)$ & $110(65.3)$ & \\
Background liver disease & & $40(23.5)$ & \\
None & $167(68.9)$ & $2(1.2)$ & \\
Fatty liver, steatosis & $58(24.1)$ & $1(0.6)$ & $<0.001$ \\
Fibrosis & $3(1.2)$ & $11(6.7)$ & $<0.001$ \\
Cirrhosis & $1(0.4)$ & $5(2.7)$ & \\
Sinusoidal dilatation & $9(3.7)$ & $420(150-475)$ & \\
Combination or other & $4(1.7)$ & $433(75-5,344)$ & \\
Median duration of operation, min & $348(120-475)$ & & \\
Median intra-operative blood loss, ml & $350(80-1,450)$ & & \\
\hline
\end{tabular}

Figures in parentheses indicate range or percentages.

\section{Safety of AIA Technique}

There were 3 incidences of staple gun misfiring in the AIA group, with one requiring suture ligation of the pedicle for intra-operative bleeding from the staple line. In 5 cases, bile leak at the staple line was identified intra-operatively, which required careful suturing with no further problems.

\section{Morbidity}

There was no statistical difference in pre- and postoperative liver function tests between the two approaches. Morbidity was similar between the groups, with the majority of complications being Clavien grades 1 and 2 [10] which were managed conservatively (table 3).

\section{Bile Leaks}

There were a total of six bile leaks; two were in the AIA and four were in the EHD group. There was one minor bile leak in each group, both of which settled promptly post-operatively. Two further leaks in the EHD group required ERCP and stenting to achieve resolution. A further patient in each group developed a leak refractory to stenting, and both required surgical reconstruction. Unfortunately, the patient in the EHD died subsequent to the reconstruction due to uncontrolled sepsis.

\section{Mortality}

The postoperative mortality for both groups was similar, with a 90 -day mortality of $2.5 \%$ for the AIA group and $4.1 \%$ for the EHD group (table 3).

\section{Oncological Outcome}

We have reported disease burden based on the number and size of metastases reported by the histopathologist rather than those seen radiologically.

The R0 resection rate (defined as the absence of microscopic tumour at the margin) was $95 \%$ for the AIA group and $94 \%$ for EHD. The median follow-up time for all patients was 30 months (range 4-126). In that time, there was an equal incidence of recurrence of disease (both intra- and extra-hepatic) of 30 and $32 \%$ in the AIA and EHD groups, respectively. There was no difference in 1-, 3- and 5 -year survival between the groups, with 5 -year survival of $37.1 \%$ in the AIA group and $37.6 \%$ for EHD (table 3 ).

\section{Discussion}

This retrospective, non-randomised study is neither designed nor powered to prove superiority of one technique over another. We have, however, presented a substantial experience of a technique that, although first 
Table 2. Details of primary, metastasis and operation

\begin{tabular}{|c|c|c|c|}
\hline & $\operatorname{AIA}(n=242)$ & $\operatorname{EHD}(n=169)$ & $\mathrm{p}$ value \\
\hline Median number of metastases & $2(1-20)$ & $2(2-20)$ & \\
\hline Distribution of metastases & & & 0.225 \\
\hline Right side only & $187(77.2)$ & $126(74.7)$ & \\
\hline Both sides & $55(22.8)$ & $41(24.1)$ & \\
\hline Caudate & 0 & $2(1.2)$ & \\
\hline Median size of largest lesion, $\mathrm{mm}$ & $40(20-180)$ & $40(20-200)$ & \\
\hline \multicolumn{4}{|l|}{ Histology of hepatectomy margin } \\
\hline R0 & $230(95)$ & $159(94)$ & \\
\hline $\mathrm{R} 1$ & $12(5)$ & $10(6)$ & \\
\hline \multicolumn{4}{|l|}{ Dukes stage of primary } \\
\hline A & $7(2.9)$ & $9(5.3)$ & \\
\hline $\mathrm{B}$ & $72(29.9)$ & $55(32)$ & \\
\hline $\mathrm{C} 1$ & $133(55.2)$ & $82(48.2)$ & \\
\hline $\mathrm{C} 2$ & $23(9.5)$ & $19(11.2)$ & \\
\hline \multicolumn{4}{|l|}{ Extent of resection } \\
\hline Anatomical & $157(64.9)$ & $73(43.2)$ & \\
\hline Anatomical + non-anatomical & $46(19.0)$ & $18(10.6)$ & \\
\hline Extended anatomical + non-anatomical & $39(16.1)$ & $78(46.2)$ & \\
\hline
\end{tabular}

Figures in parentheses indicate range or percentages.

described more than 50 years ago, has failed to gain widespread popularity. We would suggest that for specifically selected cases where the tumour mass lies an adequate distance from the porta hepatis $(>1-2 \mathrm{~cm})$, an anterior approach to controlling the main right portal pedicle, or indeed its branches (as dictated by the anatomy and the 'thickness' of the tissue of the main portal pedicle), within the liver substance can potentially avoid iatrogenic injury to the left-sided structures and may save operating time. However, use of this technique does involve a reliance on mechanical stapling devices, and these devices must be used correctly and appropriately. It has been our experience that attempting to staple tissue that is too bulky for the jaws of the device or attempting to compress too great a width of tissue within a single firing is fraught and often requires further remedial steps to oversew the pedicle which would otherwise bleed or leak bile. There were no long-term sequelae in patients who required reinforcement of the staple line, and it is our practice to apply a vascular clamp to the pedicle prior to removing a stapler that has felt subjectively to be 'tight' during the firing and to reinforce the staple line with a continuous suture prior to removing the clamp. Close attention to detail in the operation of the device, including its limitations, has been rewarded with fewer biliary complications when using the AIA
Table 3. Morbidity, mortality and recurrence

\begin{tabular}{lccc}
\hline & AIA & EHD & $\begin{array}{l}\text { p } \\
\text { value }\end{array}$ \\
& & & 0.146 \\
Post-operative complications (Clavien grade) & & \\
$\quad$ None & 172(71.1) & $110(65)$ & \\
$\quad$ Grade 1 and 2, conservative & & & \\
$\quad$ management & $61(25.2)$ & $43(25.4)$ & \\
$\quad$ Grade 3 & $2(0.8)$ & $7(4)$ & \\
$\quad$ Grade 4a & $4(1.6)$ & $5(2.9)$ & \\
$\quad$ Grade 4b & $3(1.2)$ & $4(2.4)$ & \\
30-day mortality & $4(1.7)$ & $5(3)$ & 0.786 \\
90-day mortality & $6(2.5)$ & $7(4.1)$ & 0.155 \\
Liver recurrence to date & & & \\
$\quad$ No & $169(70)$ & $116(68.6)$ & \\
$\quad$ Yes & $73(30)$ & $53(31.4)$ & \\
1-year survival, \% & 90.5 & 93.5 & \\
3-year survival, \% & 60.9 & 55.5 & \\
5-year survival, \% & 37.1 & 37.6 & \\
\hline
\end{tabular}

Figures in parentheses indicate percentages.

and none that could be directly attributed to use of the stapler itself. In both groups, the number of biliary complications fell well within the expected range compared to other series $[11,12]$. 
The lower operating time and blood loss observed in the AIA group will be partly attributable to the time saved in avoiding an EHD, but also reflects the more difficult transection required for tumours located in close proximity to the porta and an increased requirement for additional non-anatomical resections within the EHD group. There is no doubt that patients undergoing resection by way of EHD had a more challenging tumour load and distribution, and certainly selection bias exists in those suitable for AIA.

Correct selection of the most appropriate technique for the individual tumour location and patient anatomy is clearly crucial. Nonetheless, where anatomical constraints allow, we would suggest that the AIA confers certain advantages over EHD with no evidence of drawback in terms of excess morbidity or mortality, and we would advocate its use wherever possible.

\section{Conclusion}

This work suggests that the AIA for right hepatectomy is as safe as EHD and can be used in the majority of patients undergoing right hepatectomy. It has an equivalent mortality and morbidity rate, and can possibly be performed more expeditiously and with advantages in terms of parenchymal preservation. The choice of procedure will depend to a large extent on a surgeon's preference and training, but we would suggest that the ability to perform this technique should be an essential component of the liver surgeon's repertoire.

\section{References}

1 Tung TT, Quang ND: A new technique for operating on the liver. Lancet 1963;281:192193.

2 Tung TT, Quang ND: Segmentary hepatectomy by transparenchymatous vascular ligation (in French). Presse Med 1965;73:30153017.

$\checkmark 3$ Lin TY, Chen KM, Liu TK: Total right hepatic lobectomy for primary hepatoma. Surgery 1960;48:1048-1060.

4 Lortat-Jacob JL, Robert HG, Henry C: Case of right segmental hepatectomy. Mem Acad Chir (Paris) 1952;78:244-251.

$\checkmark 5$ Hodgson WJ, DelGuercio LR: Preliminary experience in liver surgery using the ultrasonic scalpel. Surgery 1984;95:230-234.
6 Fong Y, Blumgart LH: Useful stapling techniques in liver surgery. J Am Coll Surg 1997; 185:93-100.

7 Cresswell AB, Welsh FK, John TG, Rees M: Evaluation of intrahepatic, extra-Glissonian stapling of the right porta hepatis vs classical extrahepatic dissection during right hepatectomy. HPB (Oxford) 2009;11:493-498.

$\checkmark 8$ Cresswell AB, Welsh FK, Rees M: A diagnostic paradigm for resectable liver lesions: to biopsy or not to biopsy? HPB (Oxford) 2009; 11:533-540.

9 Rees M, Plant G, Wells J, Bygrave S: One hundred and fifty hepatic resections: evolution of technique towards bloodless surgery. Br J Surg 1996;83:1526-1529.
$>10$ Dindo D, Demartines N, Clavien PA: Classification of surgical complications: a new proposal with evaluation in a cohort of 6,336 patients and results of a survey. Ann Surg 2004;240:205-213.

11 Halazun KJ, Al-Mukhtar A, Aldouri A, et al: Right hepatic trisectionectomy for hepatobiliary diseases: results and an appraisal of its current role. Ann Surg 2007;246:1065-1074.

12 Karanjia ND, Lordan JT, Quiney N, Fawcett WJ, Worthington TR, Remington J: A comparison of right and extended right hepatectomy with all other hepatic resections for colorectal liver metastases: a ten-year study. Eur J Surg Oncol 2009;35:65-70. 